\title{
Local Lattice Instability Analysis on Amorphous Metals: Switching between Stable and Unstable Atoms *
}

\author{
Masaomi NISHIMURA ${ }^{* *}$, Kisaragi YASHIRO ${ }^{* * *}$ and Masahiro ARAI** \\ ${ }^{* *}$ Department of Mechanical Systems Engineering, Shinshu University \\ 4-17-1 Wakasato, Nagano 380-8553, Japan \\ E-mail: nishimu@shinshu-u.ac.jp \\ *** Graduate School of Engineering, Kobe University \\ 1-1 Rokkodai, Nada, Kobe 657-8501, Japan
}

\begin{abstract}
It is revealed that amorphous metals have many "unstable" atoms even at the equilibrium state, by local lattice instability analysis (LLIA) which discusses the positive definiteness of atomic elastic stiffness coefficients, $B_{i j}^{\alpha}$. We have explored for relationships between the deformation and these unstable atoms in inhomogeneous or disordered structure. In the present study, we have discussed the changes in unstable atoms of $\operatorname{det} B_{i j}^{\alpha}<0$ in four monatomic amorphous metals, $\mathrm{Ni}, \mathrm{Cu}, \mathrm{Zr}$ and $\mathrm{Al}$, during uniaxial tension. First, we have separately evaluated the atomic stress on stable and unstable atoms. Unstable atoms feel hydrostatic compression in the amorphous $\mathrm{Ni}, \mathrm{Cu}$ and $\mathrm{Zr}$, while they feel hydrostatic tension in the $\mathrm{Al}$ at the initial state before loading. Under the uniaxial tension, it is considered from the comparison of stress components on each stable and unstable atoms that the local stress reduction occurs by the transition of stable $\rightarrow$ unstable in $\mathrm{Ni}, \mathrm{Cu}$ and $\mathrm{Zr}$, and unstable $\rightarrow$ stable in Al. Then we have picked up atoms that have actually switched between stable and unstable. Even at the equilibrium state, so many atoms switch their stabilities while the ratios of the negative and positive change nearly balance at each moment. Both positive and negative switching are activated by rises of the structural relaxation under the tension. Moreover, there is no difference in the stress between positive and negative switching atoms while constant $\operatorname{det} B_{i j}^{\alpha}<0$ and $\operatorname{det} B_{i j}^{\alpha}>0$ atoms show different stress. We have concluded that the stress relaxation is not caused by a straightforward image of "stabilization" or "destabilization", but by "shuffle of atomic arrangement" which involves positive and negative switching simultaneously. In fact, we have observed many incidences of positive and negative stability-switching at the locally deformed area.
\end{abstract}

Key words : Local Lattice Instability Analysis, Molecular Dynamics Simulation, Elastic Stiffness Coefficients, Amorphous Metal, Stability Switching

\section{Introduction}

Amorphous metals have short and middle range order such as an icosahedral atomiccluster $^{(1)-(3)}$. These atomic scale structure play important roles in the formability and deformability of amorphous metals. Some molecular dynamics simulations suggest that the convergence of these local structural changes causes the global deformation such as a shear band $^{(4),(5)}$.

We have attempted to reveal the local and global deformation mechanism of amorphous metals by the local lattice instability analysis (LLIA) ${ }^{(6)}$ that discusses the positiveness of atomic elastic stiffness coefficients, $B_{i j}^{\alpha}$, or the second-order derivatives of atomic energy composition. On single-crystal metals which have no "unstable" atoms at the equilibrium state, the nucleation of lattice defects such as dislocation and cleavage cracking can be predicted by

${ }^{*}$ Received 18 June, 2010 (№. 10-0257) [DOI: 10.1299/jmmp.4.1563]

Copyright (C) 2010 by JSME 
$\operatorname{LLIA}^{(6),(7)}$. After the plastic deformation, unstable atoms remain as "defects", or dislocation cores and grain boundaries, in the polycrystalline metals. On the other hand, amorphous metals have many unstable atoms even at the equilibrium state ${ }^{(7)}$. We have discussed about the relationships between the deformation and these unstable atoms in the inhomogeneous or disordered structure. In our previous reports ${ }^{(8),(9)}$, the physical meanings of the unstable atoms are shown in the various simulations of amorphous and nano-polycrystalline nickels.

In the present study, we target the several monatomic amorphous metals based on the embedded atom method (EAM) or the Lenard-Jones (LJ) potential, in order to find out general characteristics of amorphous metals. Monatomic amorphous metals are made in periodic cells by using usual melt-quench simulation, and subjected to uniaxial tension. Then we evaluate the stabilities of all atoms by LLIA during the tension. First, we have separately evaluated the stresses acting on the stable and unstable atoms. Then, we have picked up atoms that have actually switched their atomic coefficients, $B_{i j}^{\alpha}$, between stable and unstable for each time interval.

\section{Local lattice instability analysis}

Wang et al. have proposed the evaluation of lattice stability at finite strain and temperature, on the basis of the positive definiteness of elastic stiffness coefficients ${ }^{(10),(11)}$. The elastic stiffness, or stress-strain, coefficients are written as ${ }^{(12)}$

$$
B_{i j k l} \equiv\left(\frac{\partial \sigma_{i j}}{\partial \varepsilon_{k l}}\right)=C_{i j k l}+\frac{1}{2}\left(\sigma_{i l} \delta_{j k}+\sigma_{j l} \delta_{i k}+\sigma_{i k} \delta_{j l}+\sigma_{j k} \delta_{i l}-2 \sigma_{i j} \delta_{k l}\right),
$$

where $\delta_{i j}$ is Kronecker's delta. The stress, $\sigma_{i j}$, and the elastic coefficients, $C_{i j k l}$, are defined as

$$
\sigma_{i j}=\frac{1}{V}\left(\frac{\partial U}{\partial \eta_{i j}}\right), C_{i j k l}=\frac{1}{V}\left(\frac{\partial^{2} U}{\partial \eta_{i j} \partial \eta_{k l}}\right) .
$$

Here $U$ is the internal energy and $V$ is the volume of crystal at the equilibrium. Note that the differentiation in Eq. (2) is for the infinitesimal virtual strain, $\eta_{i j}$, at the equilibrium state under the external load. The $B_{i j k l}$ combines the stress and actual strain, $\varepsilon_{i j}$, from the load-free reference state. In the linear elasticity region, $B_{i j k l}$ is identical to $C_{i j k l}$ but it is not equivalent in the nonlinear elasticity or finite strain region. That is, $B_{i j k l}$ represents the gradient at the stress-strain surface in the six dimensional strain space, whether the system is in the linear or nonlinear elasticity. Thus we can easily imagine the physical meaning of the Wang's stability criteria; it is the point where the crystal loses the deformation resistance. The symmetric part of the tensor of Eq. (1), $B_{i j k l}^{\mathrm{sym}} \equiv\left(B_{i j k l}+B_{l k j i}\right) / 2$, dominates the lattice stability ${ }^{(10),(11)}$. The symmetric tensor $B_{i j k l}^{\mathrm{sym}}$ is represented by the $6 \times 6$ matrix, $B_{i j}(i, j=1 \sim 6)$, in the Voigt notation $^{(12)}$. Thus the instability criterion could be written as $\operatorname{det} B_{i j}<0$.

In the EAM potential, the total energy of system, $E_{\text {tot }}$, is expressed by

$$
E_{\mathrm{tot}}=\sum_{\alpha}^{N} F\left(\rho^{\alpha}\right)+\frac{1}{2} \sum_{\alpha}^{N} \sum_{\beta(\neq \alpha)}^{N} \phi\left(r^{\alpha \beta}\right)
$$

where $F$ represents the many body term which is a functional of local density at atom $\alpha, \rho^{\alpha}$, and the $\phi$ does the 2 body term between atom $\alpha$ and $\beta$ separated with the scalar distance $r^{\alpha \beta}$. Considering the differentiation of the potential form of Eq. (3), the atomic stress, $\sigma_{i j}^{\alpha}$, and the atomic elastic coefficients, $C_{i j k l}^{\alpha}$, can be derived as

$$
\begin{aligned}
\sigma_{i j}^{\alpha}= & \frac{1}{\Omega} \sum_{\beta(\neq \alpha)}^{N}\left\{F_{\alpha}^{\prime} \rho_{\alpha \beta}^{\prime}+\frac{1}{2} \phi_{\alpha \beta}^{\prime}\right\} \frac{r_{i}^{\alpha \beta} r_{j}^{\alpha \beta}}{r^{\alpha \beta}}, \\
C_{i j k l}^{\alpha}= & \frac{1}{\Omega}\left[F_{\alpha}^{\prime \prime}\left\{\sum_{\beta(\neq \alpha)}^{N} \rho_{\alpha \beta}^{\prime} \frac{r_{i}^{\alpha \beta} r_{j}^{\alpha \beta}}{r^{\alpha \beta}}\right\}\left\{\sum_{\gamma \neq \alpha)}^{N} \rho_{\alpha \gamma}^{\prime} \frac{r_{k}^{\alpha \gamma} r_{l}^{\alpha \gamma}}{r^{\alpha \gamma}}\right\}\right. \\
& +\sum_{\beta(\neq \alpha)}^{N} F_{\alpha}^{\prime}\left\{\rho_{\alpha \beta}^{\prime \prime}-\frac{\rho_{\alpha \beta}^{\prime}}{r^{\alpha \beta}}\right\} \frac{r_{i}^{\alpha \beta} r_{j}^{\alpha \beta} r_{k}^{\alpha \beta} r_{l}^{\alpha \beta}}{\left(r^{\alpha \beta}\right)^{2}}
\end{aligned}
$$




$$
\left.+\frac{1}{2} \sum_{\beta(\neq \alpha)}^{N}\left\{\phi_{\alpha \beta}^{\prime \prime}-\frac{\phi_{\alpha \beta}^{\prime}}{r^{\alpha \beta}}\right\} \frac{r_{i}^{\alpha \beta} r_{j}^{\alpha \beta} r_{k}^{\alpha \beta} r_{l}^{\alpha \beta}}{\left(r^{\alpha \beta}\right)^{2}}\right],
$$

where $\Omega$ is the atomic volume, $r_{i}^{\alpha \beta}$ is the $i$-component of the position vector from atom $\alpha$ to $\beta$. $F_{\alpha}, \rho_{\alpha \beta}$ and $\phi_{\alpha \beta}$ denote $F\left(\rho^{\alpha}\right), \rho\left(r^{\alpha \beta}\right)$ and $\phi\left(r^{\alpha \beta}\right)$, respectively, and the prime indicates the derivative of each function. Thus we can calculate the atomic elastic stiffness coefficients, $B_{i j k l}^{\alpha}$, at any time and configuration in the simulation, by using Eqs. (1), (4) and (5). The symmetric part of $B_{i j k l}^{\alpha}$ is also used for stability analysis, so that we denote the local instability condition as $\operatorname{det} B_{i j}^{\alpha}<0(i, j=1 \sim 6)$, according to the Voigt notation.

In the system based on only the 2 body interaction such as LJ potential, the total energy of the system is expressed only with the second term of Eq. (3),

$$
E_{\mathrm{tot}}=\frac{1}{2} \sum_{\alpha}^{N} \sum_{\beta(\neq \alpha)}^{N} \phi\left(r^{\alpha \beta}\right)
$$

Thus, $\sigma_{i j}^{\alpha}$ and $C_{i j k l}^{\alpha}$ are also written as the terms that relate to the 2 body interaction in Eqs. (4) and (5),

$$
\sigma_{i j}^{\alpha}=\frac{1}{2 \Omega} \sum_{\beta(\neq \alpha)}^{N} \phi_{\alpha \beta}^{\prime} \frac{r_{i}^{\alpha \beta} r_{j}^{\alpha \beta}}{r^{\alpha \beta}}, C_{i j k l}^{\alpha}=\frac{1}{2 \Omega} \sum_{\beta(\neq \alpha)}^{N}\left\{\phi_{\alpha \beta}^{\prime \prime}-\frac{\phi_{\alpha \beta}^{\prime}}{r^{\alpha \beta}}\right\} \frac{r_{i}^{\alpha \beta} r_{j}^{\alpha \beta} r_{k}^{\alpha \beta} r_{l}^{\alpha \beta}}{\left(r^{\alpha \beta}\right)^{2}} .
$$

\section{Simulation procedure}

Monatomic amorphous metals are made by usual melt-quench simulation under the periodic boundary condition in all directions. We made four amorphous models of $\mathrm{Ni}, \mathrm{Al}, \mathrm{Cu}$ and $\mathrm{Zr}$. The interatomic potentials of $\mathrm{Ni}$ and $\mathrm{Al}$ are the EAM of Voter and $\mathrm{Chen}^{(13)}$, and those of $\mathrm{Cu}$ and $\mathrm{Zr}$ are the $\mathrm{LJ}$ of Kobayashi et al. ${ }^{(14)}$. The total number of atoms is 108,000 in each amorphous metal. These amorphous metals are then subjected to tension by elongating the cubic cell. A small strain increment of $1.0 \times 10^{-7}$ is applied on the cell in the $z$-direction at every $\Delta t=1 \mathrm{fs}$, where $\Delta t=1 \mathrm{fs}$ is the time increment of molecular dynamics calculation, resulting in the strain rate of $1.0 \times 10^{8} / \mathrm{s}$. The atom position is normalized by the cell length so that the distance of each atom is uniformly expanded in the loading process. The cell length in the transverse directions is also controlled to cancel the normal stress originated by the Poisson's contraction. The temperature is kept at $10 \mathrm{~K}$ by velocity scaling during the tension. We evaluate the stabilities of all atoms by LLIA at each $\Delta \varepsilon_{z z}=0.0005$, or $\Delta t=5000 \mathrm{fs}$, during the tension.

\section{Results and discussion}

\subsection{Stress-strain curves}

Figure 1 indicates relationships between the stress and strain of each amorphous metal under tension. Open circles in the figure indicate the peak of each stress-strain curve during tension until $\varepsilon_{z z}=0.08$. Amorphous $\mathrm{Cu}$ and $\mathrm{Zr}$ show the largest initial gradient, and the $\mathrm{Al}$ shows the smallest one. All amorphous metals show nonlinear curves from the very beginning of the loading, even though they seem to have "linear elasticity" at a glance. Then these curves alternate increases and decreases after $\varepsilon_{z z}=0.02$ except the Al. The steepest stress-drop occurs after the peak stress shown with open circles in $\mathrm{Ni}, \mathrm{Cu}$ and $\mathrm{Zr}$. The stress oscillation of $\mathrm{Al}$ is very small and the flow stress is also very low, about $0.3 \mathrm{GPa}$. Thus, the stress-strain curve of $\mathrm{Al}$ does not have a clear peak point and does not show remarkable stress-drop after the maximum stress. 


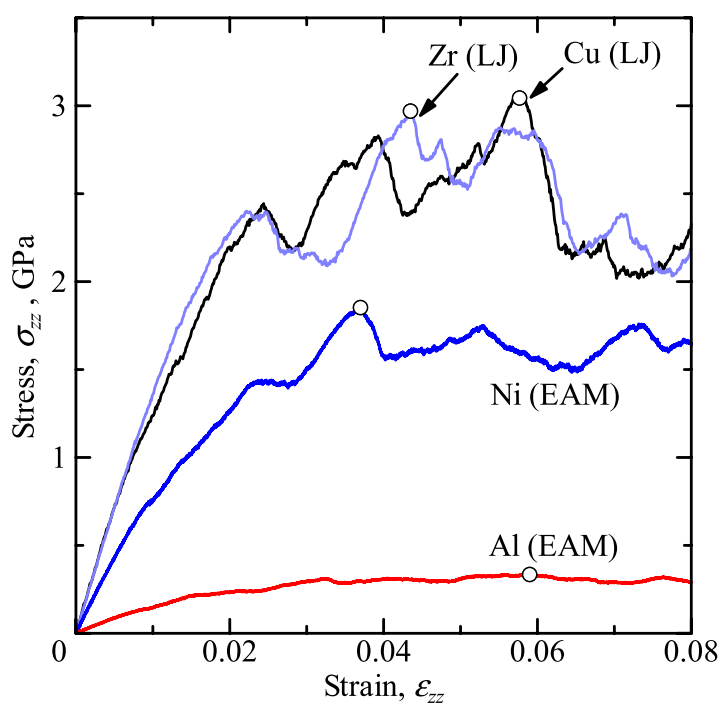

Fig. 1 Stress-strain curves.

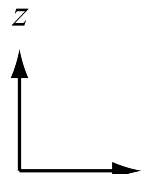

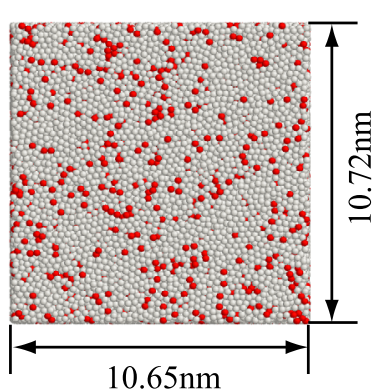

(a) Amorphous nickel

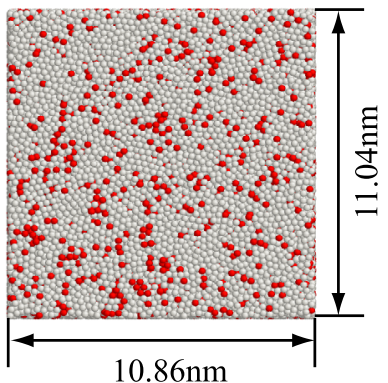

(c) Amorphous copper

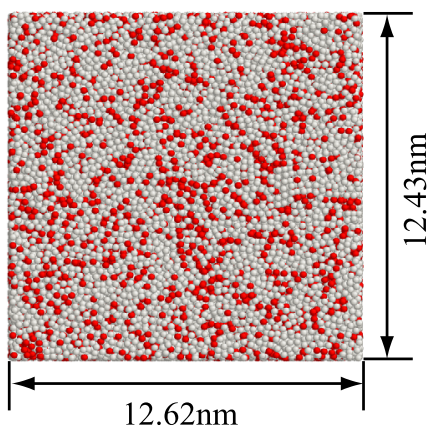

(b) Amorphous aluminum

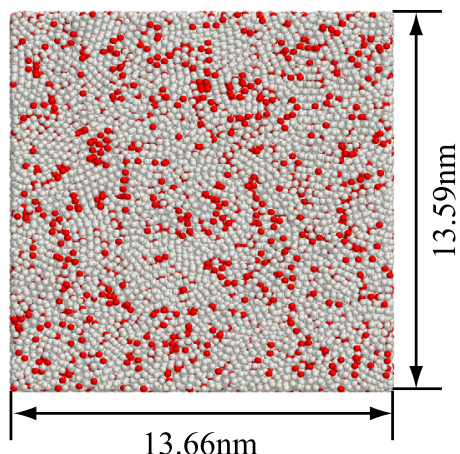

(d) Amorphous zirconium

Fig. 2 Snapshots of unstable (red) and stable (white) atoms at the initial state before loading. 


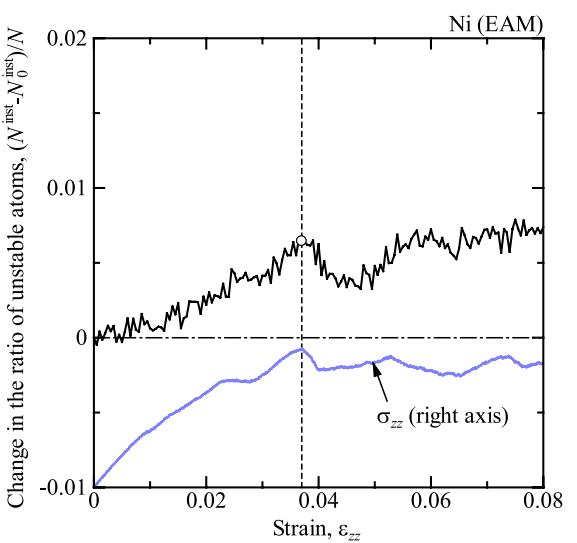

(a) Amorphous nickel

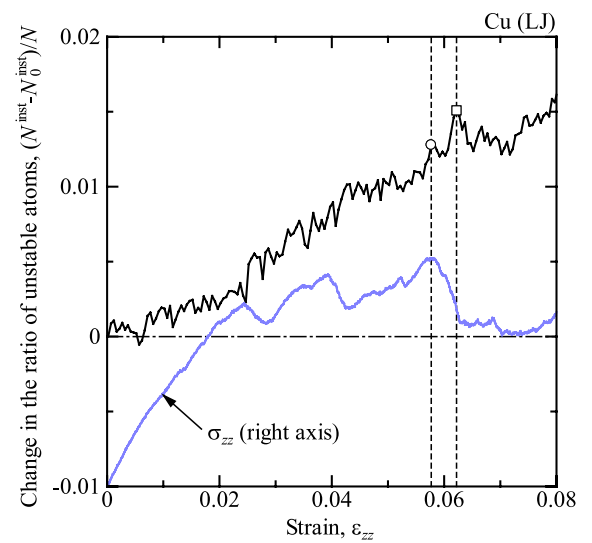

(c) Amorphous copper

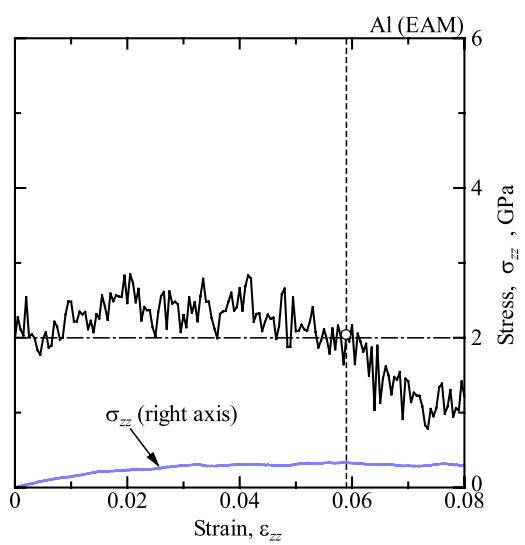

(b) Amorphous aluminum

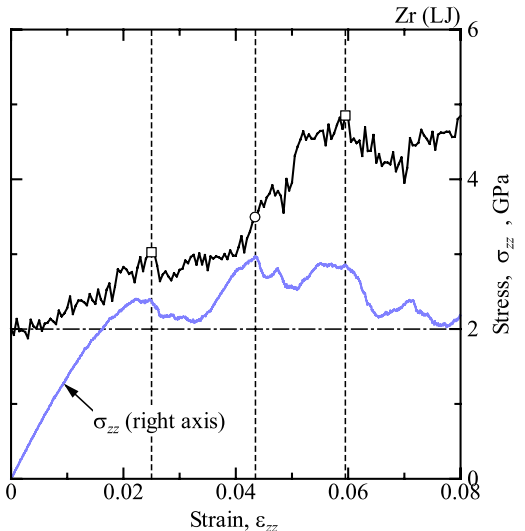

(d) Amorphous zirconium

Fig. 3 Changes in the ratio of unstable atoms from initial states, and stress-strain curves.

\subsection{Ratio and average stress of unstable atoms}

Figure 2 shows snapshots of atoms in monatomic amorphous metals at the equilibrium state before loading. The red circles are "unstable" atoms of $\operatorname{det} B_{i j}^{\alpha}<0$ while the white indicates stable ones. The equilibrium cell lengths indicated in the figure are varied from initial value to obtain the stress-free condition in each atomic radius. Thus the amorphous $\mathrm{Zr}$ has the largest size. The slight anisotropy of the lengths is also caused in the melt-quench relaxation. Amorphous metals contain many unstable atoms even in the equilibrium state but the contrast of stable and unstable is different with the atom type. The amorphous $\mathrm{Al}$ shows the highest ratio of unstable atoms about $25 \%$, while the $\mathrm{Ni}, \mathrm{Cu}$ and $\mathrm{Zr}$ have the ratio of $13 \%$, $14 \%$ and $16 \%$, respectively.

Figure 3 shows the changes in the ratio of unstable atoms under tension. For comparison, the initial ratio before tension is set to zero. Stress-strain curves are shown again in this figure, and open circles correspond to the peak point of these. All amorphous metals show subtle increase of unstable atoms at the initial stage of the loading, $\varepsilon_{z z}<0.02$. However, the increase is stopped in the amorphous $\mathrm{Al}$ at $\varepsilon_{z z}=0.02$, and then the ratio of unstable atoms decreases as shown in Fig. 3(b). After the stress-strain peak point shown with open circle, the $\mathrm{Al}$ has fewer unstable atoms than the initial state. The ratio of unstable atoms in amorphous Ni shows a peak around $\varepsilon_{z z}=0.037$ in Fig. 3(a). This peak coincides with the strain of stress peak in Fig. 1 , as shown with open circle. After this peak, the ratio shows small drop but it increases again after $\varepsilon_{z z}=0.05$. Changes in the unstable atom in the amorphous $\mathrm{Cu}$ and $\mathrm{Zr}$ do not coincide with open circle, or peak point of stress-strain, while they have peaks as denoted with open squares. Both of open squares in $\mathrm{Zr}$, around $\varepsilon_{z z}=0.025,0.059$, are located at the edge of 


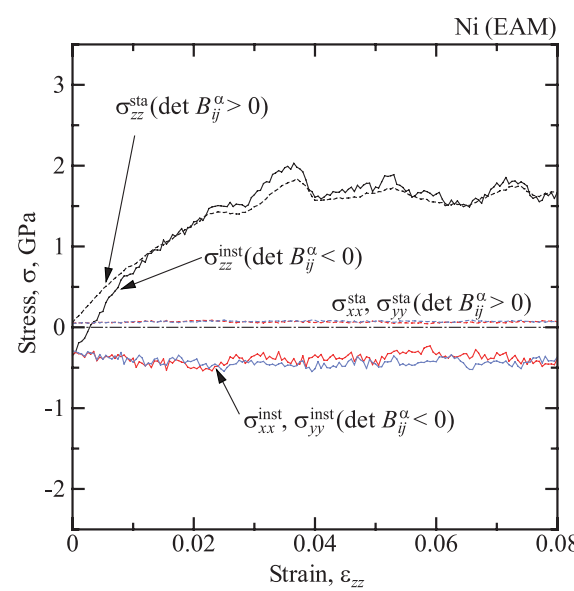

(a) Amorphous nickel

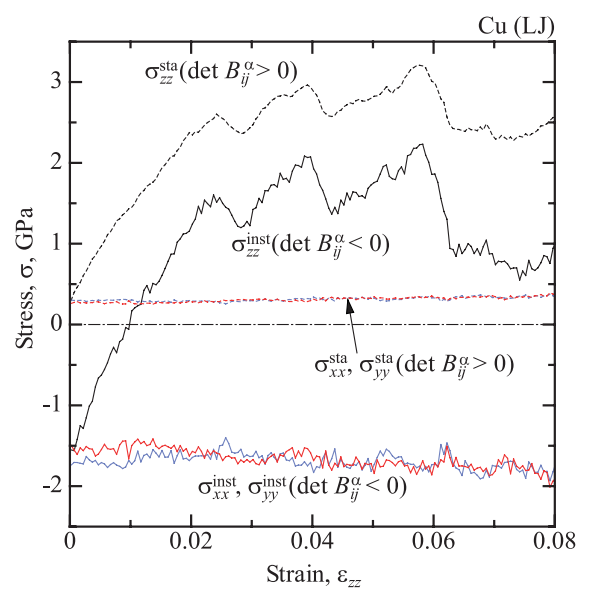

(c) Amorphous copper

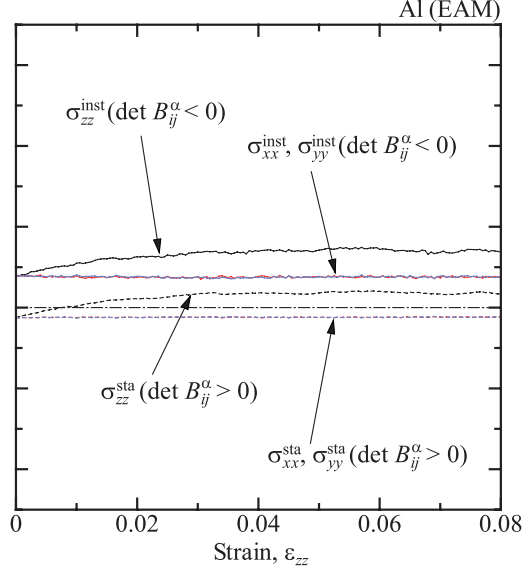

(b) Amorphous aluminum

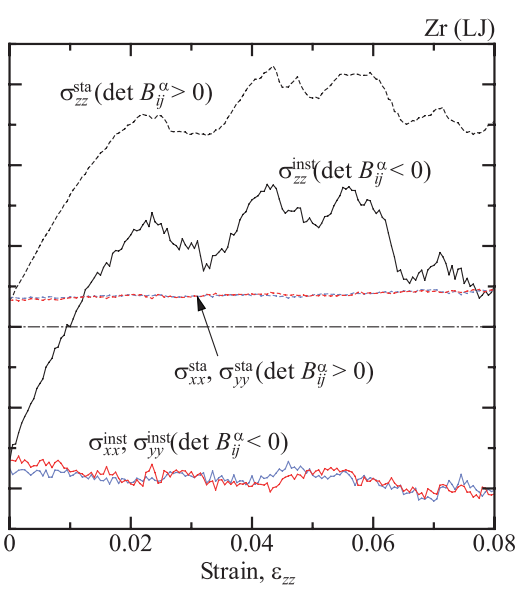

(d) Amorphous zirconium

Fig. 4 Changes in the stresses acting on stable and unstable atoms.

"cliff" of the stress-drop. On the other hand, the open square in $\mathrm{Cu}$ is located on the way of the "cliff". The amorphous $\mathrm{Cu}$ shows the largest ratio-change about 0.016 by the tension up to $\varepsilon_{z z}=0.08$, while this change is fractional less than 2,000 atoms.

We separately evaluate the stress on each atom and average it for stable and unstable atoms as $\sigma_{i j}^{\text {sta }}$ and $\sigma_{i j}^{\text {inst }}$, respectively. Figure 4 shows the changes in the normal components of the $\sigma_{i j}^{\text {sta }}$ and $\sigma_{i j}^{\text {inst }}$. At the initial state before loading, the values at $\varepsilon_{z z}=0$ in the figure, unstable atoms feel hydrostatic compression in the amorphous $\mathrm{Ni}, \mathrm{Cu}$ and $\mathrm{Zr}$ while they feel hydrostatic tension in the Al. Stresses on stable atoms show opposite signs against unstable ones because the system stresses, or averages of all atoms, are controlled to zero in the equilibrium state. Under the tension, magnitude relations between $\sigma_{i j}^{\text {sta }}$ and $\sigma_{i j}^{\text {inst }}$ do not change except the $\sigma_{z z}$ in the $\mathrm{Ni}$, where the stress difference in $\sigma_{z z}^{\text {sta }}$ and $\sigma_{z z}^{\text {inst }}$ vanishes at $\varepsilon_{z z}>0.012$ in Fig. 4(a). The role of unstable atoms in this amorphous $\mathrm{Ni}$ have been detailed in our previous report ${ }^{(9)}$. As previously shown in Fig. 3, the unstable atoms increase in amorphous $\mathrm{Ni}, \mathrm{Cu}$ and $\mathrm{Zr}$ during tension. Of course there is a large difference between the number of stable/unstable atoms, the average stress of unstable atoms is always lower than that of stable ones in the lateral direction $(\mathrm{Ni}, \mathrm{Cu}, \mathrm{Zr})$. It is also true for the tensile direction in $\mathrm{Cu}$ and $\mathrm{Zr}$. That is, the transition from stable to unstable leads the local stress reduction in all directions ( $\mathrm{Cu}$ and $\mathrm{Zr}$ ) or only in the lateral direction (Ni). In contrast with these amorphous metals, the stresses on unstable atoms in the $\mathrm{Al}$ are always higher than those on stable ones. However, as shown in Fig. 3 the unstable $\rightarrow$ stable transition takes place in the tension of $\mathrm{Al}$, and this causes the local stress 


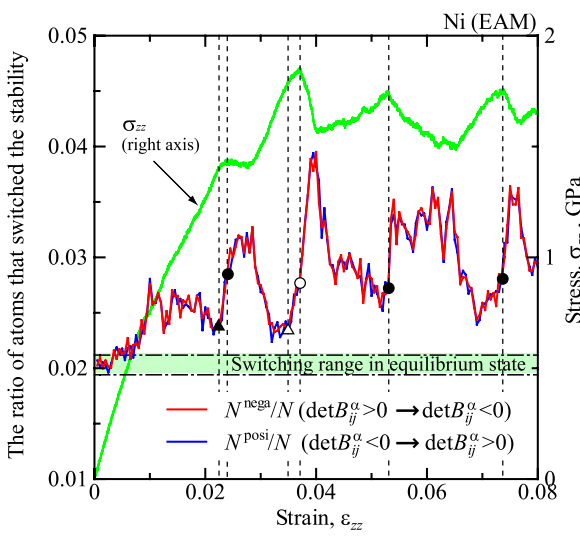

(a) Amorphous nickel

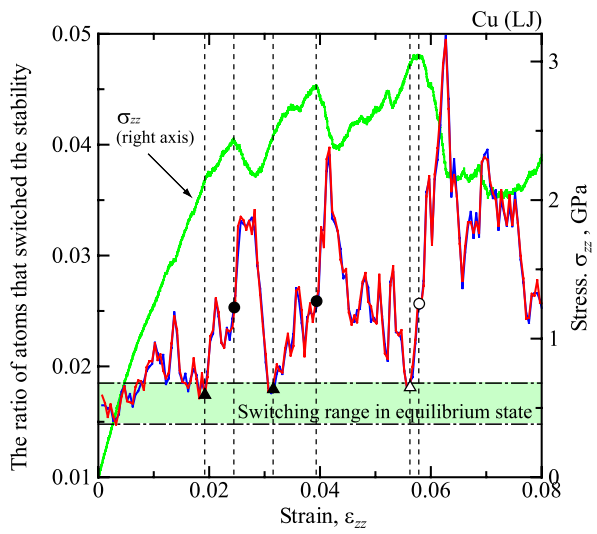

(c) Amorphous copper

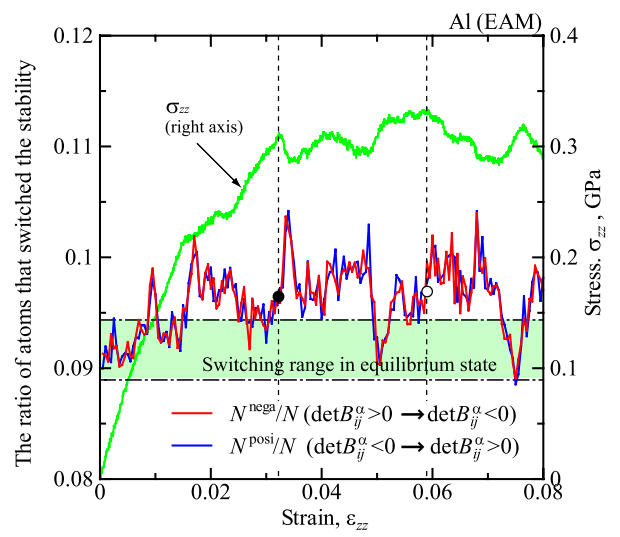

(b) Amorphous aluminum

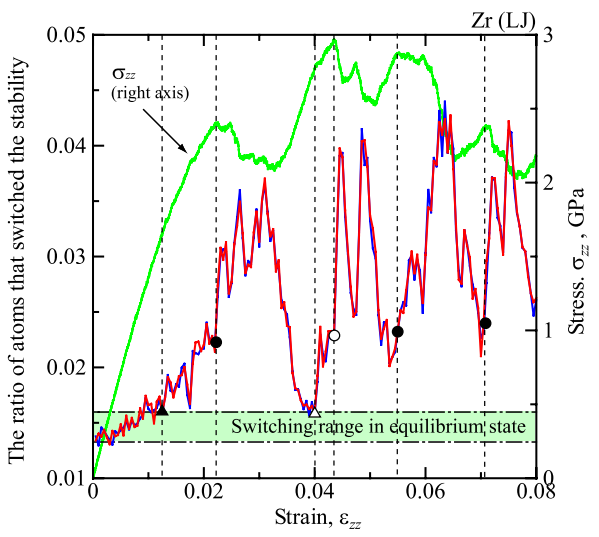

(d) Amorphous zirconium

Fig. 5 Changes in the ratio of atoms that have switched their $\operatorname{det} B_{i j}^{\alpha}$ between positive and negative for each interval of $\Delta \varepsilon_{z z}=0.0005$, and stress-strain curves.

decrease in all directions. All in all, the local stress decrease occurs by the transition of stable $\rightarrow$ unstable $(\mathrm{Ni}, \mathrm{Cu}, \mathrm{Zr})$ and unstable $\rightarrow$ stable $(\mathrm{Al})$.

\subsection{Stable $\leftrightarrow$ unstable switching at each moment}

Then we have picked up atoms that have actually switched between stable and unstable for each interval of $\Delta \varepsilon_{z z}=0.0005$. Figure 5 shows ratios of these atoms, where $N^{\text {posi }} / N$ and $N^{\text {nega }} / N$ are the ratio of atoms with the positive change from $\operatorname{det} B_{i j}^{\alpha}<0$ to $\operatorname{det} B_{i j}^{\alpha} \geq 0$ and the negative change from $\operatorname{det} B_{i j}^{\alpha} \geq 0$ to $\operatorname{det} B_{i j}^{\alpha}<0$, respectively. We have also evaluated the ratio of atoms that have switched the stability for each time interval of $\Delta t=5000 \mathrm{fs}$ in the equilibrium state without tension, where $\Delta t$ correspond to the time for extending $\Delta \varepsilon_{z z}=0.0005$ in this tensile simulation. The upper and lower limits of the switching ratio are monitored during $100,000 \mathrm{fs}$ in the equilibrium and indicated by colored zones between horizontal dash-dotted lines. For comparison, the stress-strain curves are shown again in this figure. The scale of $\sigma_{z z}$ is changed for each atom type to remark the up and down of stress-strain response, as indicated in the right axis. At a glance both changes of $N^{\text {posi }}$ and $N^{\text {nega }}$ coincides with each other; however, there is subtle difference and its accumulation leads the global changes of unstable atoms previously shown in Fig. 3. In amorphous metals, so many atoms switch their stability, or their positive definiteness of $\operatorname{det} B_{i j}^{\alpha}$, while the ratio of the negative and positive switches nearly balances at each time interval. The stable $\leftrightarrow$ unstable switching at each interval is largest in the $\mathrm{Al}$, about one fifth of total atoms are switched as can be seen in the left axis of Fig. 5(b). Open circles in the figure denote the strain point of the maximum stress peak in Fig. 1. The circles except Al locate on the middle of steep positive slopes in Fig. 5. The steep 


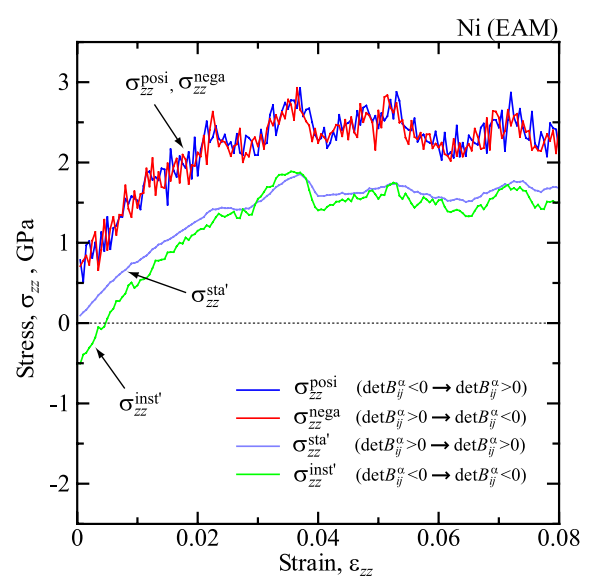

(a) Amorphous nickel

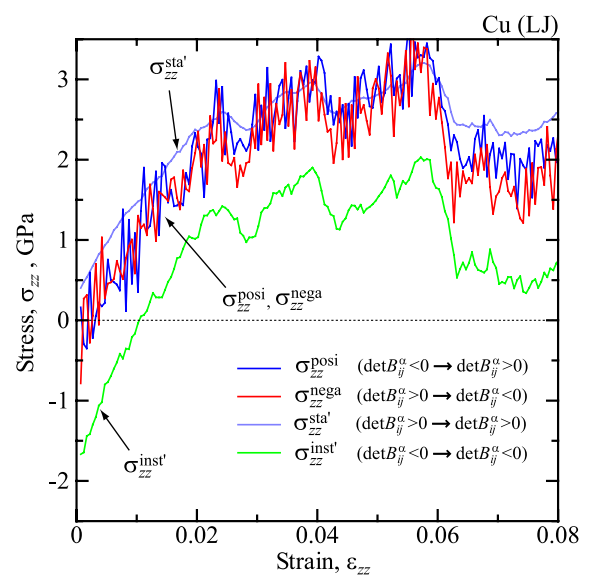

(c) Amorphous copper

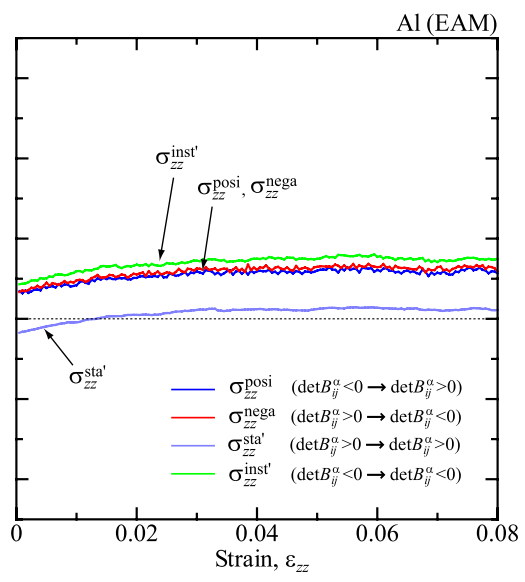

(b) Amorphous aluminum

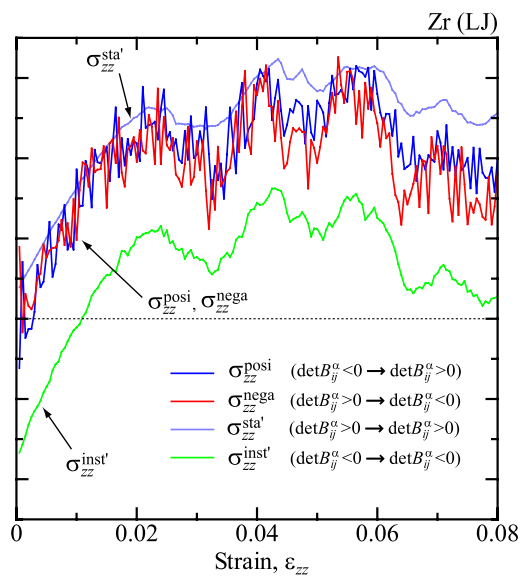

(d) Amorphous zirconium

Fig. 6 Changes in averaged stresses for each type of the stability switching.

slopes of $N^{\text {posi }}$ and $N^{\text {nega }}$ start from around the upper lines of the switching range in the noload equilibrium, as shown open triangles. Here, the stability switching under the equilibrium state is caused by the subtle structural change by the atomic perturbation or thermal vibration. If we assume the ratio of this "noise" is not changed by tension, the drastic increase beyond the upper dash-dotted line suggests the incidence of stability switching by large structural changes such as collapses of atomic cluster shown in our previous report ${ }^{(7)}$. Closed triangles show onset points of the rise from the upper dash-dotted line, just same as open triangles. Gradients of stress-strain curves change at these triangle points, and slow down as the stability switchings increase. This suggests that the stress relaxation is caused by "shuffle of atomic arrangement" which involves positive and negative switching simultaneously. Closed circles denote another "stress maximum" of stress-strain curves. These closed circles and the open circle align in horizontal direction or same switching ratio, and these circles are located on the way of drastic increase from triangle points. These correspondences imply that the stress peak could be estimated by some critical value of stability switching, although the value might be different for each condition such as strain rate and size of cubic cell. In the amorphous $\mathrm{Al}$ of Fig. 5(b), it is difficult to find out the correspondence between the switching ratio and stress-strain response, still we can also observe the correspondence at the stress-strain peak, as shown with open and closed circle.

We also separately evaluate the atomic stress by the stability switching. Atoms are categorized into four types, $N^{\text {posi }}, N^{\text {nega }}, N^{\text {sta' }}$ and $N^{\text {inst' }}$, by the stability switching for each interval 
of $\Delta \varepsilon_{z z}=0.0005 ; N^{\text {posi }}$ and $N^{\text {nega }}$ are the number of atoms with positive/negative stabilityswitching as same in Fig. 5, while $N^{\text {sta }}$ and $N^{\text {inst' }}$ are that of stable and unstable atoms which do not switch their stability during the $\Delta \varepsilon_{z z}$. Figure 6 shows the averaged stress for each switching type, $\sigma^{\text {posi }}, \sigma^{\text {nega }}, \sigma^{\text {sta }}$ and $\sigma^{\text {inst' }}$. At the moment of stress evaluation, numbers of stable/unstable atoms in system are $N^{\text {sta }}=N^{\text {posi }}+N^{\text {sta' }}$ and $N^{\text {inst }}=N^{\text {nega }}+N^{\text {inst' }}$. Thus the stresses for stable/unstable atoms previously shown in Fig. 4 have the relationship as follows,

$$
\begin{aligned}
\sigma^{\text {sta }} & =\left(\sigma^{\text {sta }} \times N^{\text {sta }}+\sigma^{\text {posi }} \times N^{\text {posi }}\right) / N^{\text {sta }}, \\
\sigma^{\text {inst }} & =\left(\sigma^{\text {inst' }} \times N^{\text {inst' }}+\sigma^{\text {nega }} \times N^{\text {nega }}\right) / N^{\text {inst }} .
\end{aligned}
$$

Figure 6 shows the stress only in the tensile direction. The tendency of $\sigma_{z z}^{\text {sta' }}$ and $\sigma_{z z}^{\text {inst' }}$ is almost same as Fig. 4. On the other hand, the stresses on the switching atoms, $\sigma_{z z}^{\text {posi }}$ and $\sigma_{z z}^{\text {nega }}$, are thoroughly different from $\sigma_{z z}^{\text {sta }}$ neither $\sigma_{z z}^{\text {inst }}$ even though they are also "stable" and "unstable" atoms at each moment. It also should be noted that there is little difference between $\sigma_{z z}^{\text {posi }}$ and $\sigma_{z z}^{\text {nega }}$. This result is consistent with the previous conclusion that the positive and negative switching occur together as the "shuffle of atomic arrangement". That is, both atoms with positive and negative switchings feel the same stress because these are involved with the same "shuffle".

Finally, we have considered the relationship between the local deformation and the stability switching. Figure 7 and 8 show (a) the magnitude and direction of migration of each atoms with small vector and (b) the distribution of atoms that have switched between stable and unstable, during $\varepsilon_{z z}=0.035 \sim 0.037$ in amorphous $\mathrm{Ni}$ and $\varepsilon_{z z}=0.056 \sim 0.058$ in amorphous $\mathrm{Cu}$, respectively. These periods just correspond to spans from the onset point of the increment of $N^{\text {posi }}$ and $N^{\text {nega }}$, (open triangle in Fig. 5(a) and (c)), to the peak points of stress-strain curves, (open circle). The trajectory and distribution is shown only thin part in the simulation cell, where the atoms show the largest migration during these periods. Colored circles in Fig. 7(b) and 8(b) are atoms that have switched the stability only once for each fourtimes interval of $\Delta \varepsilon_{z z}=0.0005$ during these periods; green atoms have negatively switched and blue ones have positively done. Many atoms switch their stabilities more than once, but these are mainly caused by probabilistic changes such as the thermal vibration. Thus, these atoms are not shown in Fig. 7(b) and 8(b) because we want to understand the relationship between the local deformation and the stability switching. We can find remarkable atom rearrangements as shown with ellipsoids in Fig. 7(a) and 8(a). These regions have many atoms that have switched their stability in Fig. 7(b) and 8(b). This demonstrates that the "shuffle of atomic arrangement" simultaneously produce both positive and negative stability-switching. It is noteworthy that these periods are before the peaks of stress-strain curves. The emergence of these local events leads to the global deformation. It is suggested the possibility that we can evaluate the origin of global deformation by the observation of the stability switching.

\section{Conclusion}

In order to find out the general mechanism of deformation in amorphous metals by the local lattice instability analysis that discusses the positive definiteness of atomic elastic stiffness coefficients, $B_{i j}^{\alpha}$, we have discussed the changes in unstable atoms of $\operatorname{det} B_{i j}^{\alpha}<0$ in four monatomic amorphous metals during uniaxial tension. First, we have separately evaluated the atomic stress on stable and unstable atoms. Unstable atoms feel hydrostatic compression in the amorphous $\mathrm{Ni}, \mathrm{Cu}$ and $\mathrm{Zr}$, while they feel hydrostatic tension in the $\mathrm{Al}$ at the initial state before loading. If the amorphous metals are subjected to tension, unstable atoms increase in the $\mathrm{Ni}, \mathrm{Cu}$ and $\mathrm{Zr}$ while those decrease in the $\mathrm{Al}$. It is considered from the comparison of stress components on each stable and unstable atoms that the local stress reduction occurs by the transition of stable $\rightarrow$ unstable in $\mathrm{Ni}, \mathrm{Cu}$ and $\mathrm{Zr}$, and unstable $\rightarrow$ stable in $\mathrm{Al}$. Then we have picked up atoms that have actually switched between stable and unstable, and revealed the following facts : (1) so many atoms switch their stabilities even at the equilibrium state, while the ratio of the negative and positive switching nearly balances at each time interval, 


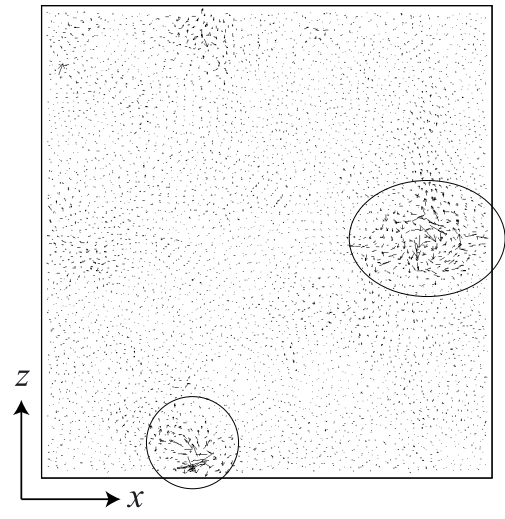

(a) Trajectories of atom motion

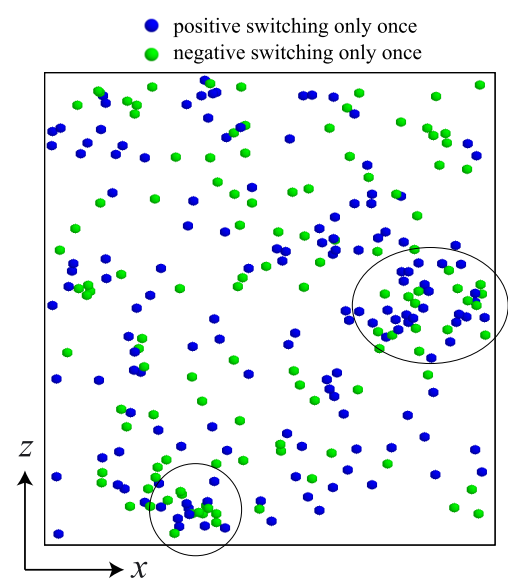

(b) The distribution of atoms that have switched between stable and unstable

Fig. 7 Comparison between atom motions and distribution of atoms that have switched the stability during $\varepsilon_{z z}=0.035 \sim 0.037$ in amorphous Ni.

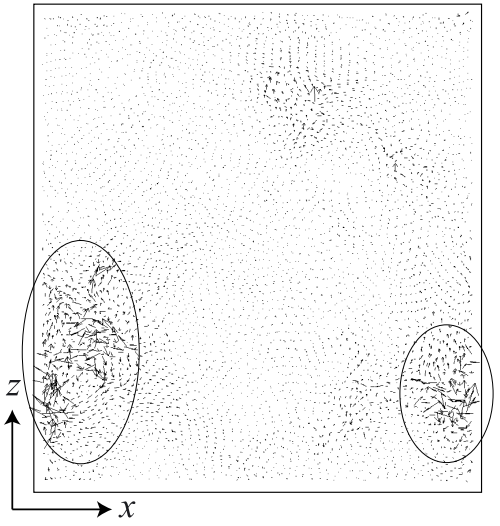

(a) Trajectories of atom motion

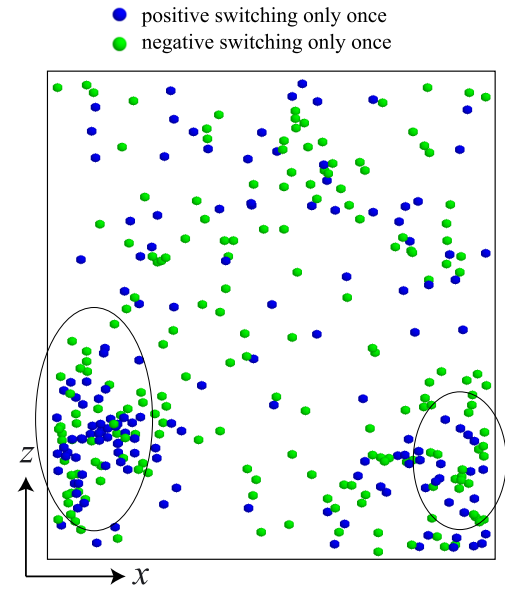

(b) The distribution of atoms that have switched between stable and unstable

Fig. 8 Comparison between atom motions and distribution of atoms that have switched the stability during $\varepsilon_{z z}=0.056 \sim 0.058$ in amorphous $\mathrm{Cu}$.

(2) gradients of stress-strain curves slow down along with rises of the stability switching, (3) changes in atomic stresses with positive and negative switching almost coincides with each other, while those of constantly stable/unstable atoms have clear difference as mentioned, and (4) the local structural relaxation, or the "shuffle of atomic arrangement", simultaneously produces both positive and negative stability-switching.

\section{References}

( 1 ) M. Shimono and H. Onodera, Short-Range and Medium-Range Order in Supercooled Liquids of Alloys, Materials Science and Engineering A, Vol.449-451, (2007), pp.717721.

( 2 ) T. Takeuchi, S. Nakano, M. Hasegawa, K. Soda, H. Sato, U. Mizutani, K. Itoh and T. Fukunaga, Local Atomic Arrangements and Electronic Structure of Zr-Ni-Al Bulk Metallic Glass -Analysis by Use of the Relevant Crystals-, Materials Science and Engineering A, Vol.449-451, (2007), pp.599-604.

( 3 ) H. W. Sheng, W. K. Luo, F. M. Alamgir, J. M. Bai and E. Ma, Atomic Packing and Shortto-Medium-Range Order in Metallic Glasses, Nature, Vol.439, No.26, (2006), pp.419- 
425.

( 4 ) S. Ogata, F. Shimizu, J. Li, M. Wakeda and Y. Shibutani, Atomistic Simulation of Shear Localization in Cu-Zr Bulk Metallic Glass, Intermetallics, Vol.14, (2006), pp.10331037.

( 5 ) M. Wakeda, Y. Shibutani, S. Ogata and J. Park, Multiple shear banding in a computational amorphous alloy model, Applied Physics A, Vol.91, (2008), pp.281-285.

( 6 ) K. Yashiro and Y. Tomita, Local lattice instability at a dislocation nucleation and motion, Journal de Physique IV, Vol.11, (2001), Pr5-3-Pr5-10.

( 7 ) K. Yashiro, M. Nishimura and Y. Tomita, Deformation analysis of amorphous metals based on atomic elastic stiffness coefficients, Modelling and Simulation in Materials Science and Engineering, Vol.14, (2006), pp.597-605.

( 8 ) K. Yashiro, M. Nishimura and Y. Tomita, Local Lattice Instability Analysis on Nanopolycrystalline and Amorphous Metals: Deformation Limit of Weak Area, Proc. of APCOM'07-EPMESC XI, (2007), MS31-5-1.

( 9 ) K. Yashiro, M. Nishimura, M. Higuchi and Y. Tomita, Local Lattice Instability Analysis on Nano-polycrystalline and Amorphous Metals: Deformation Limit of Weak Area, Proc. of ICHMM-2008, (2008), pp.308-311.

(10) J. Wang, S. Yip, S. R. Phillpot and D. Wolf, Crystal instabilities at finite strain, Physical Review Letters, Vol.71, (1993), pp.4182-4185.

(11) J. Wang, J. Li, S. Yip, S. R. Phillpot and D. Wolf, Mechanical instabilities of homogeneous crystals, Physical Review B, Vol.52, (1995), pp.12627-12635.

(12) P. C. Wallace, Thermodynamics of Crystals, Wiley, Newyork. (1972).

(13) A. F. Voter and S. P. Chen, Accurate Interatomic Potentials for Ni, $\mathrm{Al}$ and $\mathrm{Ni}_{3} \mathrm{Al}$, Materials Research Society Symposium Proceedings, (1987), pp.175-180.

(14) S. Kobayashi, K. Maeda and S. Takeuchi, Computer simulation of atomic structure of $\mathrm{Cu}_{57} \mathrm{Zr}_{43}$ amorphous alloy, Jornal of Physical Society of Japan, Vol.48, (1980), pp.11471152. 